\title{
APLICATIVO EXCEL/VBA PARA ESTIMATIVAS DE COEFICIENTES DE ATIVIDADES A DILUIÇÃO INFINITA ATRAVÉS DO MODELO MOSCED E VERIFICAÇÃO DE AZEOTROPIA DE MISTURAS BINÁRIAS
}

\author{
L. G. Oliveira, S. P. Nascimento, T. D. Romão, J. A. Cavalcante, N. A. Costa \\ Universidade Federal da Paraíba, Departamento de Engenharia Química \\ E-mail para contato: nagelalves@hotmail.com
}

\begin{abstract}
RESUMO - O presente trabalho tem por objetivo apresentar uma planilha eletrônica com programação em objeto-modelo VBA para estimativas de coeficientes de atividades a diluição infinita usando o modelo MOSCED (Modified Separation of Cohesive Energy Density) e verificação de azeotropia da mistura binária a baixas e moderadas pressões, visto que a fase vapor é considerada ideal. O programa foi codificado na forma modular. Os módulos são: a) banco de dados contendo os parâmetros do modelo de MOSCED e constantes da pressão de vapor da equação de Antoine; b) criação de caixas de diálogos para interação do usuário com o programa; c) codificações de subprogramas VBA para as estimativas dos coeficientes de atividade a diluição infinita e d) validação dos resultados fornecidos pelo programa. O resultado final é um aplicativo, para fins educacionais e/ou comerciais, capaz de fornecer estimativas dos coeficientes de atividades à diluição infinita e verificações de azeotropia na mistura com rapidez e confiabilidade.
\end{abstract}

\section{INTRODUÇÃO}

Um azeótropo é uma mistura líquida em equilíbrio com a fase vapor de igual composição. A mistura líquido-vapor apresenta comportamento de uma substância pura, vaporizando-se e condensando-se com composição, temperatura e pressão constantes. $\mathrm{O}$ conhecimento da composição e da temperatura (ou da pressão) de pontos azeotrópicos binários e multicomponentes de uma mistura é de fundamental importância para o projeto de colunas de destilação, pois estes pontos fornecem restrições adicionais quanto ao grau de separação que pode ser obtida por meio dessa operação unitária. A existência de azeotropia depende, essencialmente, de dois fatores: grau de não idealidade da mistura e diferenças entre os pontos de ebulição dos componentes. O grau de não idealidade da mistura é fornecido pelos coeficientes de atividade dos componentes à diluição infinita, que representam a não idealidade máxima de um componente em solução, pois referem-se ao limite de diluição em que se tem uma molécula de um componente rodeada unicamente por moléculas do outro componente (Azevedo, 1995). As pressões de vapor fornecem as diferenças entre os pontos de ebulição dos componentes. 


\subsection{Modelo MOSCED}

O modelo MOSCED (Modified separation of cohesive energy density) foi desenvolvido por Thomas e Eckert (1984), sendo uma extensão da teoria da solução regular de ScatchardHildebrand. Essa teoria assume que a entropia em excesso e o volume em excesso da mistura são desprezíveis, ou seja, a energia livre de Gibbs de uma solução é proporcional à diferença entre as densidades de energia coesiva da mistura e dos componentes puros.

A correlação proposta por Thomas e Eckert (1984) é dada por:

$$
\ln \gamma_{2}^{\infty}=\frac{V_{2}}{R T}\left[\left(\lambda_{1}-\lambda_{2}\right)^{2}+\frac{q_{1}^{2} q_{2}^{2}\left(\tau_{1}-\tau_{2}\right)^{2}}{\psi_{1}}+\frac{\left(\alpha_{1}-\alpha_{2}\right)\left(\beta_{1}-\beta_{2}\right)}{\xi_{1}}\right]+d_{12}
$$

onde o índice 1 é para o solvente e o índice 2 para o soluto.

Os parâmetros envolvidos na Equação (1) são:

- Volume molar dos componentes para a temperatura de $20{ }^{\circ} \mathrm{C}, V_{1}$ e $V_{2}$;

- Parâmetros de dispersão, $\lambda_{1}$ e $\lambda_{2}$;

- Parâmetro de indução, $q_{1}$ e $q_{2}$;

- Parâmetros polares, $\tau_{1}$ e $\tau_{2}$;

- Parâmetros de acidez, $\alpha_{1}$ e $\alpha_{2}$;

- Parâmetros de basicidade, $\beta_{1}$ e $\beta_{2}$;

- Parâmetro de assimetria (leva em conta o efeito resultante da diferença de polaridade, $\psi_{1}$ );

- Parâmetro que levam em conta as ligações de pontes de hidrogênio, $\xi_{1}$.

- Termo combinatorial de Flory-Hugins que leva em conta a diferença de tamanho entre as moléculas, $d_{12}: d_{12}=\ln \left(\frac{V_{2}}{V_{1}}\right)^{a a}+1-\left(\frac{V_{2}}{V_{1}}\right)^{a a}$

- Parâmetro $a a$ é definido como: $a a=(0,953-0,00968)\left(\tau_{2}^{2}-\alpha_{2} \beta_{2}\right)$, onde os parâmetros para o componente 2 são avaliados na temperatura $T$ do sistema.

Thomas e Eckert (1984) reportam os parâmetros para 144 substâncias químicas na temperatura de $20{ }^{\circ} \mathrm{C}$. Os parâmetros $\tau, \alpha, \beta, \psi, \xi$ e aa são dependentes da temperatura do sistema. Portanto, para temperaturas diferentes de $20{ }^{\circ} \mathrm{C}$, as seguintes expressões são utilizadas para correções dos mesmos:

- Parâmetros $\tau$, $\alpha$ e $\beta: \tau_{T}=\tau_{293}\left(\frac{293}{T}\right)^{0,4}, \alpha_{T}=\alpha_{293}\left(\frac{293}{T}\right)^{0,8}$ e $\beta_{T}=\beta_{293}\left(\frac{293}{T}\right)^{0,8}$

- Parâmetro $\psi: \psi=P o l+0,011 \alpha_{T} \beta_{T}$, onde $P o l=q^{4}\left[1,15-1,15 \exp \left(-0,020 \alpha_{T}^{3}\right)\right]+1$. 
- Parâmetro $\xi: \xi=0,68(P o l-1)+\left\{3,4-2,4 \exp \left[-0,023\left(\alpha_{0} \beta_{0}\right)^{1,5}\right]\right\}$, onde $t=293 / T$ (T é a temperatura em Kelvin). Os subscritos 0 e $T$ referem-se, respectivamente a temperatura de $20{ }^{\circ} \mathrm{C}$ e a temperatura do sistema $T$.

\subsection{Pressão de Vapor - Equação de Antoine}

A equação de Antoine foi escolhida para estimativa da pressão de vapor com as constantes características de cada espécie química reportadas por Yaws (2007).

$$
\log _{10} P^{s a t}=A-B /(T+C)
$$

Onde $P^{\text {sat }}$ é a pressão de vapor em mmHg. $A, B$ e $C$ são constantes características de cada espécie de química e $T$ é a temperatura em Celsius.

\subsection{Verificação de Azeotropia}

A verificação de azeótropo, em sistemas binários, é realizada através da definição de uma grandeza denominada volatilidade relativa (Para pressões baixas e moderadas).

$$
\alpha_{12}=\frac{y_{1} / x_{1}}{y_{2} / x_{2}}=\frac{\gamma_{1} P_{1}^{s a t}}{\gamma_{2} P_{2}^{s a t}}
$$

onde $y_{i}$ e $x_{i}$ são as frações molares da espécie $\mathrm{i}$, respectivamente, na fase vapor e na fase líquida. $P_{i}^{\text {sat }}$ e $\gamma_{i}$ são, respectivamente, a pressão de vapor e o coeficiente de atividade da espécie $i$.

No equilíbrio líquido-vapor normal, a volatilidade relativa pode ser maior ou menor que a unidade em toda a faixa de composição. Os seus valores extremos ocorrem nos limites de composição. Nestes limites, tem-se que:

- Limite inferior: Para $x_{1} \rightarrow 0, x_{2} \rightarrow 1, \gamma_{1}=\gamma_{1}^{\infty}$ e,$\gamma_{2}=1 ; \operatorname{logo},\left(\alpha_{12}\right)_{x_{1}=0}=\frac{\gamma_{1}^{\infty} P_{1}^{\text {sat }}}{P_{2}^{\text {sat }}}$

- Limite superior: Para $x_{1} \rightarrow 1, x_{2} \rightarrow 0, \gamma_{1}=1$ e $\gamma_{2}=\gamma_{2}^{\infty} ; \operatorname{logo},\left(\alpha_{12}\right)_{x_{1}=1}=\frac{P_{1}^{s a t}}{\gamma_{2}^{\infty} P_{2}^{s a t}}$

Para desvios positivos em relação à lei de Raoult, $\gamma_{i}>1$ e assim, $\left(\alpha_{12}\right)_{x_{1}=0} \geq 1$. Portanto, existirá azeótropo sempre que $\left(\alpha_{12}\right)_{x_{1}=1} \leq 1$, pois a volatilidade relativa é uma função contínua da composição $x_{1}$ e deverá passar pela unidade para alguma composição $0<x_{1}<1$. Assim, para a existência de azeótropo, as seguintes desigualdades devem ser satisfeitas:

$$
\frac{\gamma_{1}^{\infty} P_{1}^{\text {sat }}}{P_{2}^{\text {sat }}} \geq 1 \text { e } \frac{P_{1}^{\text {sat }}}{\gamma_{2}^{\infty} P_{2}^{\text {sat }}} \leq 1
$$


Para desvios negativos em relação à lei de Raoult, $\gamma_{i}<1 \mathrm{e}$, assim, $\left(\alpha_{12}\right)_{x_{1}=1} \geq 1$. Logo, existirá azeótropo sempre que $\left(\alpha_{12}\right)_{x_{1}=0} \leq 1$, pois a volatilidade relativa é uma função contínua da composição $x_{2}$ e deverá passar pela unidade para alguma composição $0<x_{2}<1$. Assim, para existir azeótropo, as seguintes desigualdades devem ser satisfeitas:

$$
\frac{\gamma_{1}^{\infty} P_{1}^{\text {sat }}}{P_{2}^{\text {sat }}} \leq 1 \text { e } \frac{P_{1}^{\text {sat }}}{\gamma_{2}^{\infty} P_{2}^{\text {sat }}} \geq 1
$$

\section{METODOLOGIA}

A metodologia adotada para o desenvolvimento dos subprogramas JAVA é esquematizada na Figura 1.

Figura 1 - Metodologia para o desenvolvimento do aplicativo

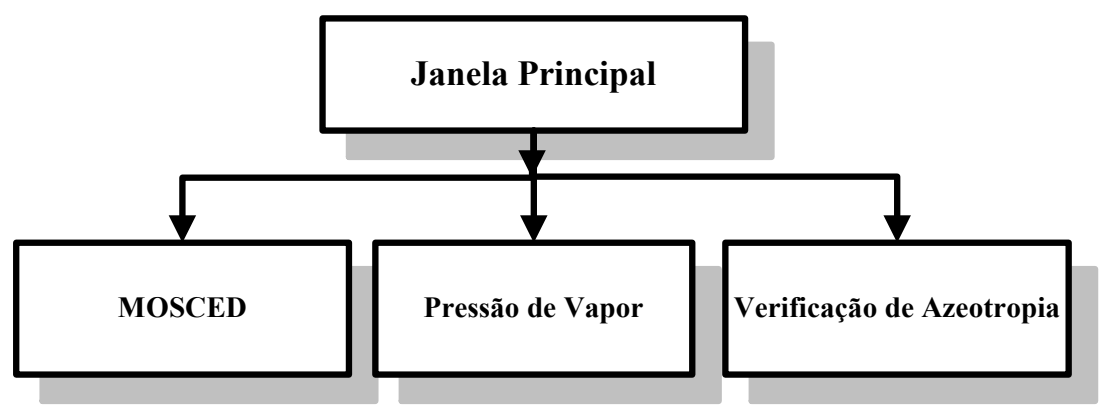

\subsection{Esquema do desenvolvimento do aplicativo}

O desenvolvimento do aplicativo principia-se com a criação do banco de dados de 108 espécies químicas contendo as constantes características de cada espécie do modelo MOSCED reportadas por Thomas e Eckert (1994) e as constantes características da equação de Antoine reportadas por Yaws (2007). Na Figura 2 é apresentada a planilha utilizada para iniciar as macros codificadas no VBA.

Figura 2 - Planilha utilizada para a configuração (cálculos) e saída de dados

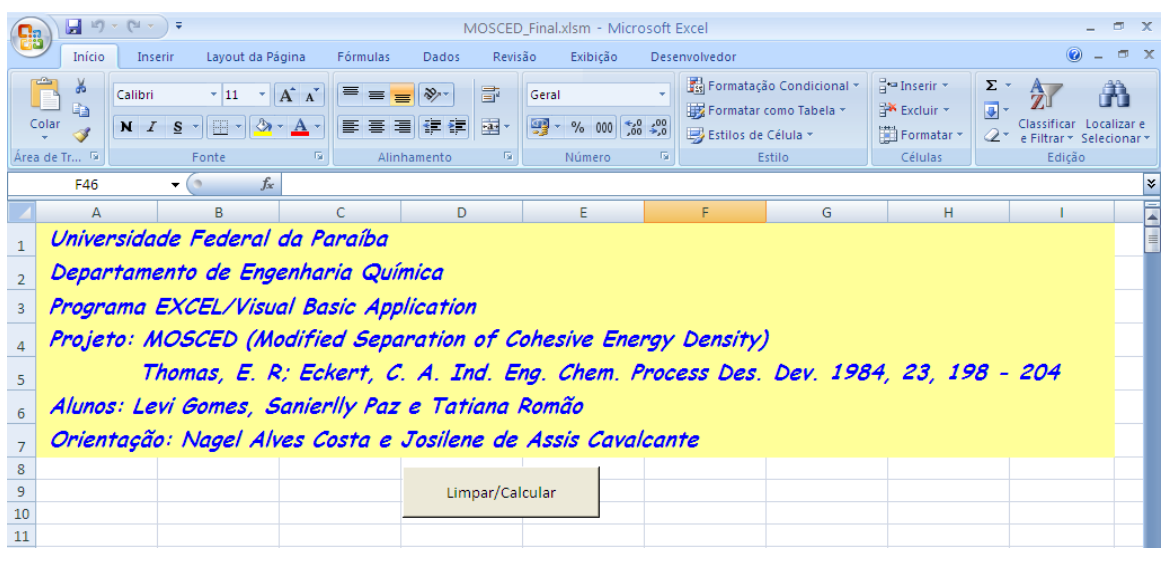


Nas Figuras 3 e 4 são mostradas as caixas de diálogo que permitem ao usuário escolher, respectivamente, o tipo de cálculo e os componentes presentes na mistura binária.

Figura 3 - Escolha do tipo de cálculo

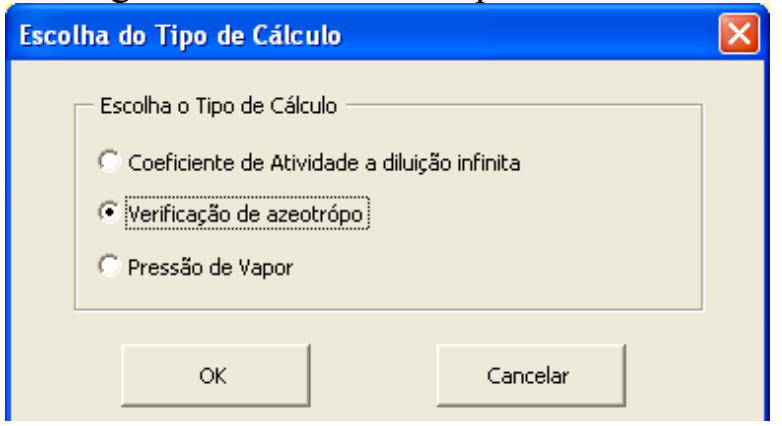

Figura 4 - Escolha das espécies

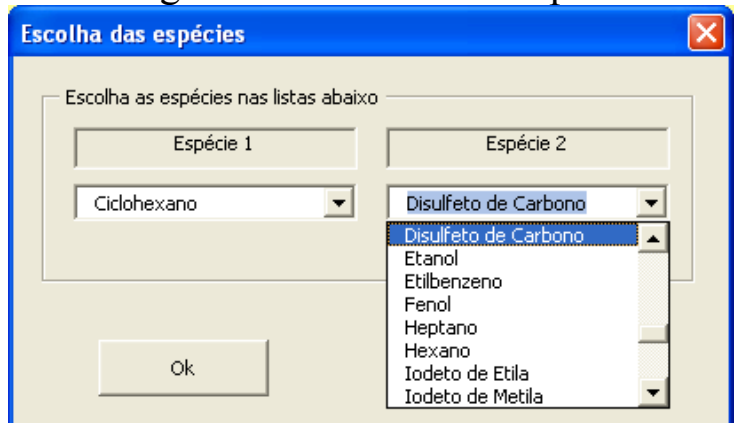

Se a escolha dos componentes estiver fora da ordem decrescente de volatilidade, o programa utiliza uma subrotina de ordenação por contagem proposta por Knuth (1999). Por exemplo, as espécies químicas da Figura 4 são ordenadas para fornecer: a) componente 1 (mais volátil) - CS2 e b) componente 2 (menos volátil) - Ciclohexano.

A especificação da temperatura é mostrada na Figura 5.

Figura 5 - Entrada de dados

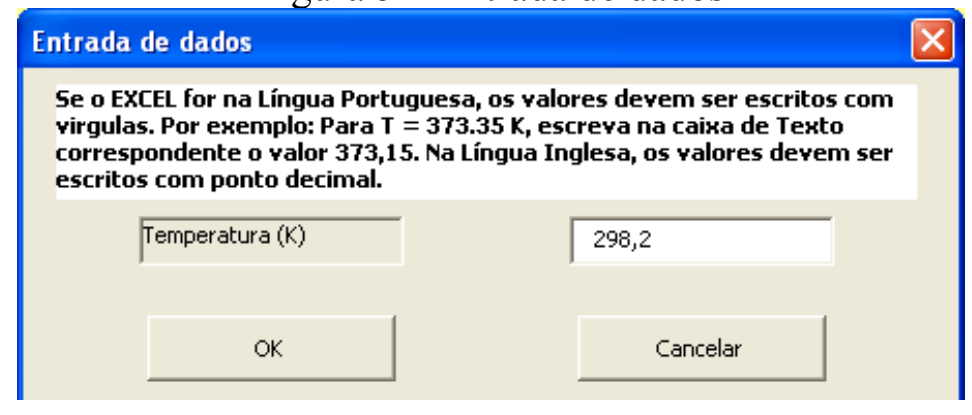

\section{VALIDAÇÃO DO APLICATIVO}

Vários sistemas reportados por Thomas e Eckert (1984) foram utilizados para a validação do aplicativo proposto. Alguns resultados são apresentados na Tabela 1.

Tabela 1 - Coeficiente de atividade a diluição infinita

\begin{tabular}{l|l|c|c|c|c}
\hline \multirow{2}{*}{ Soluto $\left(\gamma_{1}^{\infty}\right)$} & \multirow{2}{*}{ Solvente } & \multirow{2}{*}{$\mathrm{T}(\mathrm{K})$} & \multicolumn{2}{c|}{ Thomas e Eckert (1984) } & \multirow{2}{*}{ Aplicativo } \\
\cline { 4 - 5 } & & & Exp & Mosced & \\
\hline Ciclohexano & $\mathrm{CS} 2$ & 298,2 & 1,59 & 1,48 & 1,483 \\
Acetonitrila & $\mathrm{CCl} 4$ & 293,2 & 13,4 & 11,83 & 11,714 \\
Acetona & CCl4 & 303,9 & 3,03 & 2,83 & 2,834 \\
Hexano & Clorofórmio & 319,8 & 1,79 & 1,94 & 1,938 \\
Trietilamina & Clorofórmio & 323,0 & 0,27 & 0,12 & 0,116 \\
Hexano & Metanol & 298,2 & 27,00 & 26,82 & 26,791 \\
Clorofórmio & Nitrometano & 293,2 & 2,20 & 2,42 & 2,418 \\
Hexano & Nitrometano & 298,2 & 48,00 & 42,20 & 42,148 \\
\hline
\end{tabular}


O programa foi exaustivamente testado para a verificação dos resultados fornecidos. As pequenas diferenças que se verificam na Tabela 1 entre os resultados calculados através do aplicativo com os reportados por Thomas e Eckert (1984) são devido às diferenças entre as precisões numéricas utilizadas no aplicativo e pelos pesquisadores, que reportaram os resultados com duas casas decimais.

A partir dos valores calculados pelo aplicativo dos coeficientes de atividade a diluição infinita e das pressões de vapor das espécies presentes na mistura binária foi possível verificar que a mesma apresenta ou não azeotropia, ou seja, se as desigualdades fornecidas pelas equações (5) e (6) são satisfeitas. Os resultados para vários sistemas binários são apresentados na Tabela 2.

Tabela 2 - Verificação de azeotropia pelo aplicativo para os sistemas binários

\begin{tabular}{l|l|c|c|c|c|c|c}
\hline \multicolumn{1}{c|}{ Espécie 1 } & Espécie 2 & $\mathrm{T}(\mathrm{K})$ & $\gamma_{1}^{\infty}$ & $\gamma_{2}^{\infty}$ & $\left.\alpha\right|_{x 1=0}$ & $\left.\alpha\right|_{x 1=1}$ & Azeótropo? \\
\hline CS2 & Ciclohexano & 298,2 & 1,289 & 1,483 & 4,975 & 2,603 & Não \\
CC14 & Acetonitrila & 293,2 & 8,149 & 11,714 & 10,476 & 0,110 & Sim \\
Acetona & CCl4 & 303,9 & 2,834 & 2,167 & 5,674 & 0,924 & Sim \\
Clorofórmio & Hexano & 319,8 & 1,582 & 1,938 & 2,034 & 0,663 & Sim \\
Clorofórmio & Trietilamina & 323,0 & 0,251 & 0,116 & 0,775 & 26,648 & Sim \\
Metanol & Hexano & 298,2 & 58,479 & 26,791 & 49,619 & 0,032 & Sim \\
Clorofórmio & Nitrometano & 293,2 & 2,418 & 3,141 & 13,872 & 1,826 & Não \\
Hexano & Nitrometano & 298,2 & 42,148 & 38,611 & 178,899 & 0,110 & Sim \\
\hline
\end{tabular}

\section{CONCLUSÃO}

Os resultados indicaram que a planilha eletrônica com programação VBA fornece estimativas com confiabilidade e rapidez para o coeficiente de atividade a diluição infinita, verificação de azeotropia e pressão de vapor. As pequenas diferenças que se verificam nos cálculos mostrados ao longo do trabalho são devido às diferenças entre as precisões numéricas utilizadas pelos autores e o aplicativo.

\section{REFERÊNCIAS}

AZEVEDO, E. G. Termodinâmica Aplicada. Escolar Editora, 1995.

KNUTH, D. E. Art of Computer Programming - V.3 - Sorting And Searching. Addison Wesley. Segunda Edição, 1998.

THOMAS, E. R; ECKERT, C. A. Prediction Activity Coefficients by a Modified Separation of Cohesive Energy Density Model and UNIFAC. Ind. Eng. Chem. Process Des. Dev. 1984, 23, $198-204$.

YAWS, C. L. The Yaws Handbook of Vapor Pressure. Gulf Publishing Company, 2007. 